\title{
Improvement of ZigBee Using by Thread and Backpressure Algorithm
}

\author{
Negar Jadidkar ${ }^{a^{*}}$, Hossein Samimi ${ }^{b}$ \\ ${ }^{a}$ Department of Computer Engineering, Science and Research, Islamic Azad university, Tehran, Iran \\ ${ }^{b}$ Assistant Professor, Iran Telecom Research Center (ITRC), Tehran, Iran
}

Received: 06 March 2017; Accepted: 15 June 2017; Published: 08 September 2017

\begin{abstract}
In wireless sensor networks, two approaches of tree and mesh routing are introduced to determine the path of packets during the transition process. Tree routing is a simple routing protocol with low overhead that in this protocol father-child bonds for packet transmission from the source to the destination is used. The biggest problem of routing is the increase of the number of mutations in comparison with other routing protocols. In order to improve this problem, protocols have been introduced in recent years to determine a shortcut path on the basis of the tree routing. This study is an attempt to analyse and evaluate the existing routing algorithms, identify and overcome their disadvantages, also in some other protocols, only reducing the number of mutations has been discussed. However, to achieve this goal leads to increased energy consumption and thus reducing the lifetime of the network; reducing the number of mutations is an important parameter and can reduce delays in the network, however, it should be noted the energy consumption in ZigBee networks is a very important debate. Besides that, this study will try - in addition to reducing the average number of mutationsto reduce the traffic load near the root node in the proposed algorithms. As a result, on the one hand, the application of this algorithm in ZigBee networks reduces delays and on the other hand, will also lead to balancing of load and energy in the network. Using this algorithm, the scope and lifetime of the proposed protocol-based networks can be increased.
\end{abstract}

Index Terms: ZigBee network 2- tree routing 3- reducing the delay 4- energy balance 5- performance evaluation.

(C) 2017 Published by MECS Publisher. Selection and/or peer review under responsibility of the Research Association of Modern Education and Computer Science

\footnotetext{
* Corresponding author.

E-mail address:
} 


\section{Introduction}

ZigBee is a powerful communication standard for wireless personal networks and is founded based on the physical layer and Media Access Control on standardization IEEE802.15.4 and has defined network and application layers as well as security services on it and for devices with low power consumption, there are low cost and high reliability and based on these characteristics, ZigBee Union has developed its application in many fields such as smart energy, health, telecommunications, and sales services. Tasks such as dynamic network, address allocation, routing and network management is on the shoulders of the network layer in the standard of ZigBee, which is in the heart of this standard. ZigBee backs more than 64,000 nodes in the network, each node in a network ZigBee is assigned a unique 16-bit short address in a distributed manner. In these networks two routing protocols are used in which the system or the user can choose their type of routine based on the system applications. The response protocol used in ZigBee mesh networks is AODVjr. This protocol is a simplified version of the protocol AODV which is used in ad hoc networks; these types of protocols are the ones based on the need, that is, every time a source node wants to send data to a destination node, a route discovery process takes place; therefore, without a doubt, overhead of route discovery process and memory usage will increase consistent with traffic and the number of sessions in the network.

Another routing which is used in ZigBee is tree routing, which does not have the overhead associated with this process in terms of memory usage and bandwidth due to the use of distributed addressing scheme and not using the route discovery process. In this routing, each node by comparing the hierarchical destination address with the address of its parent or children can understand to the data to which of them without doing any route discovery operation.

The biggest advantage of this routing is that every node in network without route discovery operations can send data to any other node in the network. Because of this feature, TR is a promising protocol for devices with limited memory and processing which are used in smart, Internet-based networks of objects. However, data are transported in a tree structure in TR, even if the destination is in the neighbourhood of source, this means the routing does not provide the optimal route for data transmission. Yet solutions have been provided to reduce the number of steps in this algorithm based on the neighbouring relationships, but since the shortest route is not necessarily the best route, and due to the importance of energy efficiency in network in order to increase its lifetime, in this thesis using data from a neighbouring table and inserting some parameters in it and using the neighbouring relationships, in the selection of the next step, several parameters were considered simultaneously and using a smart way in the selection of the next step, the study tries to partly resolve this problem. The aim of this thesis is to modify the performance of the protocol and provide a smart way to choose the next step in a data transfer from an original or central node to the destination.

\section{Tree Routing Protocol}

In this algorithm, a node with a depth of $d$ and the address of $A_{m}$ determines its next mutation to send the packet to the destination with the address of $A_{d}$ as follows:

- If $A_{m}<A_{d}<A_{m}+C_{\text {skip }}(d-1)$, then $A_{d}$ is considered to be child of $A_{m}$.

- On the other hand, if $A_{d}>A_{m}+C_{\text {skip }} R_{m}, A_{d}$ is an ending means for the $A_{m}$ node; otherwise, the address of the next mutation will be a child of the $A_{m}$ node.

$$
A_{n}=A_{m}+C_{\text {skip }}(d) \times\left[\frac{A_{d}-A_{m}-1}{C_{\text {skip }}(d)}\right]+1
$$

The address of the next mutation for the nodes of the destination which is not a child of $A_{m}$ address of father node will be $A_{m}$ 


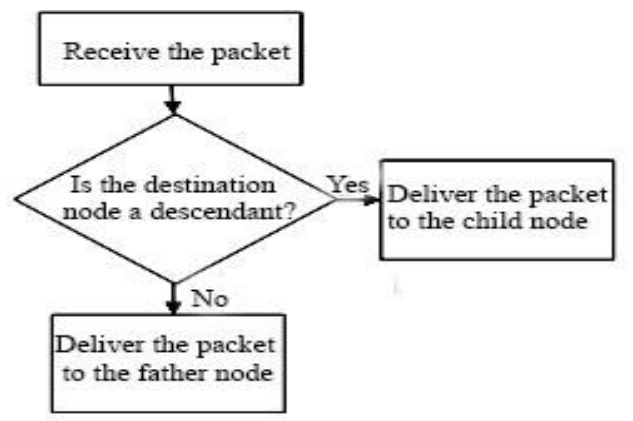

Fig.1. Tree routing protocol.

\section{The Proposed Algorithm}

In the proposed algorithm, the objective is to balance the energy consumption in the network and consequently to increase network lifetime and to reduce delays in healthy transferring of the packets to the destination. In this algorithm, an attempt is made to optimize the chosen path both in terms of energy consumption and path cost. Accordingly, following the modifications on the routing algorithm in the next step, among the neighbors of a node, we will use a threaded tree and the back-pressure algorithm to consider simultaneously three criteria: the number of remaining steps to the destination, bond quality, and residual energy of the node.

Each node upon receiving data in its neighboring table seeks the destination node, and if it finds the destination node in its neighborhood, it will send the packet to the destination node. Otherwise, each neighboring node will calculate its path cost to the destination. As mentioned earlier, this is simply possible using tree index. Then, using the threaded tree and the back-pressure algorithm with the three criteria: the number of remaining steps to destination for neighboring node, the remaining energy of the neighboring node and the quality of the bond between the source node and the neighbor node among the existing nodes available at the neighboring table, one node will be chosen as the next step.

\section{A. Design and implementation of the proposed system}

In the previous step the essentials and the elements needed to develop an appropriate system were addressed, so that this system, taking into account the objectives and constraints, could provide an appropriate output. Now it is time to weigh the implementation of the system based on what was stated in the design section.

\section{B. How to calculate inputs in the proposed system.}

After examining factors in routing and criteria for the right path in the ad-hoc networks, three quantities were selected to choose the path. Using these three quantities in the route discovery phase, the better route can be identified. The first quantity is the number of steps of the route, the quantity which indicates the number of available steps between the source node and the destination node. In the traditional DSR, the criterion for route choosing is the smaller number of steps. This quantity is inversely proportional to the suitability of a path, and in fact the value of a path. The more the number of steps are, the greater the possibility of path cancellation is due to the dynamic topology, so the path is not suitable.

The second quantity deals with the average number of neighbors of the central nodes in the path. This quantity shows the crowd and the available load along the path. The more the number of neighboring nodes on the path is, the more the possibility of congestion on that path will be. When the number of neighbors of the central nodes in the path is more, then the number of controlling packets received from the neighbors will be 
more; and on the other hand, in response to these received packets, the central node should send more packets. Overall, it can be mentioned that when the nodes have more neighbors, they will be involved in processing a great number of controlling packets; therefore, the network load will be greater on that path. So, the value of the path in which the number of neighbors of the central nodes is lower. The paths that have lower level of loads are appropriate as there is less delay in them and the possibility of dropping the packet by filling buffers of central nodes is lower in them.

The third quantity is the average of the remaining energy in the central nodes of the path. In mobile ad hoc network, in which the nodes are faced with energy constraints, energy management is vital at the network level. For this purpose, the third input quantity in the proposed system, the average of remaining energy in the central nodes of the path was chosen so that the value of path will be investigated in terms of available sufficient energy in the central nodes. The greater is the energy of a path, the wiser will be to choose the path. If the energy in the central nodes is low, with the energy being run out in one of the central nodes, the path will be eliminated and an interrupt will be caused in data transmission.

In order to obtain values for each input, the functions contained in the Protocol DSR can be used and the input quantities can be calculated with applying some changes. For this purpose, when we are looking to find a path from the source to a specific destination, the number of nodes that this path contains composes the first input of the proposed system.

To obtain the values of two other inputs, the information stored in the very central nodes is used. When a path to the intended destination is found, each node on that path calculates its remaining amount of energy as well as the number of nodes which are located in its neighborhood and finally the resulting value is divided by the number of steps of the path sot that the average number of neighbors of the central nodes of the path and the average amount of the remaining energy in these nodes are calculated.

\section{Threaded binary tree}

In a threaded binary tree in a leaf, the nodes point to the left child and right child is null. Because of not using child nodes point in the leaves, the amount of the allocated memory remains virtually unused, which these nodes point can be used to optimize the in order traversal.

Instead of using recursive algorithm, in order traversal of a non-recursive algorithm is used, which also takes less time. Also the contents of the point of left and right null nodes can be changed in a way that it points to the next and previous node point in the in order tree traversal. In this way, when it comes to a leaf node, there is no need for a recursive call to find the next node, because we have the next node address in the field of the leaf node pointer. This pointer is called thread pointer. [23]

\section{In order traversal in the threaded tree}

The traversal starts from the leftmost node, we store the value of the node, then we move to its right thread (pointer), we store the value of the new node and then move to its right subtree. After traversing by the right thread pointer, we move to the new tree. To illustrate, this is explained in figure 2.

Now, according to the Figure 3-4, if node 5 wants to send data to node 7, with the help of its right thread pointer it will move to node 6 with a leap into its parent node and will be sent to the leftmost leaf to the right side of the tree; with this threading, by two leaps the data is sent from node 5 to node 7; therefore, the traversing speed becomes faster and prevents congestion in the buffer. 


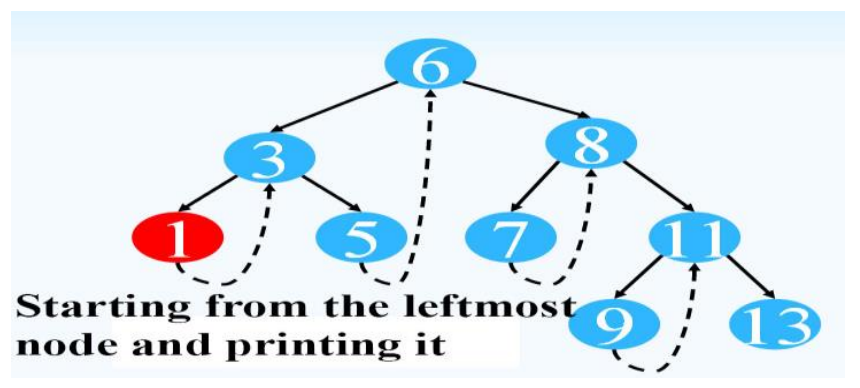

Fig.2. Tree Threading.

In this section the full dynamic routing algorithms of back pressure will be introduced and discussed. This type of routing algorithms does not calculate the paths in advance; the next step is selected dynamically. These decisions are made based on the amount or degree of crowding of neighboring nodes. When scheduling algorithm with the largest weighting the lines of communication are used, the output will be optimized. The routing of back pressure is devoted solely to the alignment networks. This algorithm can be used for ad hoc networks and wireless sensor networks. Back pressure principle can also be used to check other areas such as product assembly and network processing. This section focuses on communication networks, where the packets originated from multiple data slow flow enter and must be delivered to the appropriate destination; backpressure algorithm is performed at intervals. Any interval tries to route the data in directions where differential backlog between the neighboring nodes reaches the maximum level. This is similar to how water circulates in a network of pipes through pressure slopes. However, back-pressure algorithm can be used for multi-commodity networks and the networks whose transfer rates can be chosen from a plethora of options [23].

In our proposed model, the threading tree for high-priority data considering the amount or degree of crowding in the neighboring nodes and ordinary tree search algorithm inspired by the back-pressure algorithm will be used.

\section{Characteristics of back-pressure algorithm:}

1) The algorithm maximizes network output.

2) The algorithm is probably effective for networks that change along with time.

3) The algorithm can be implemented without data of traffic input rates or the possibility of the channel status.

In any case, the implementation of this algorithm may be difficult in networks where interference is high. Back pressure is mainly studied in theoretical frameworks. In practice, wireless networks typically have been used in implementing alternative methods of routing based on shortest way of calculations or network flooding, including routing ad hoc on demand distance vector (AODV), Geographic routing and Extremely Opportunistic Routing (EXOR) [24 and 25].

\section{E. Foundation of back-pressure algorithm}

The main back-pressure algorithm was introduced by Tassiulas and Ephremides in 1992. They studied a radio network of multi-hop packet with random packet inputs and a fixed set of options for choosing bonds. Their algorithm includes the step of choosing maximum weight bond and step of backlog differential routing. The back-pressure algorithm, designed for calculating slow flow of multi-commodity network, was developed for planning for mobile networks. The back pressure has been analyzed and combined with optimization techniques through the theory of a slow flow by Lyapunov $[24,25]$. 


\section{F. Decision and selection of the optimized commodity in back-pressure algorithm}

In each interval $\mathrm{T}$ visits the back-pressure controller $\mathrm{S}(\mathrm{t})$ and performs the following three steps:

- $\quad$ First, for each bond of (A, B), the optimized commodity chooses $C_{a b}^{o p t}(t)$.

- Then, it determines what matrix $\left(\mu_{a b}(t)\right)$ is used in $\Gamma_{S(t)}$.

- Finally, this will be the value of the commodity $C_{a b}^{o p t}(t)$.

An example has been illustrated in figure 3-5. We assume that in each line there are only two types of data:

1. Real time (high priority)

2. Non-real time (low priority)

And these are in the correct units of measurement packets. If we focus on the direct link (1 and 2 ), the backlog of the line will be:

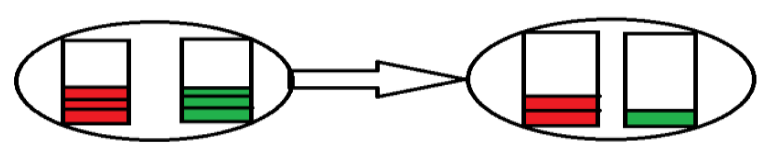

Fig.3. Line backlog.

Therefore, optimized commodity for sending 1 to 2 in non-real-time data slot T links has high priority. On the other hand, optimized commodity for sending reverse link of 2 to 1 in non-real-time data slot $\mathrm{T}$ has a low priority.

Equation [26].

$Q_{1}^{(r e d)}(t)-Q_{2}^{(r e d)}(t)=1$

$Q_{1}^{(\text {green })}(t)-Q_{2}^{(\text {green })}(t)=2$

For simplicity, we assume that the topology has only four possible options which are shown in Fig 4.

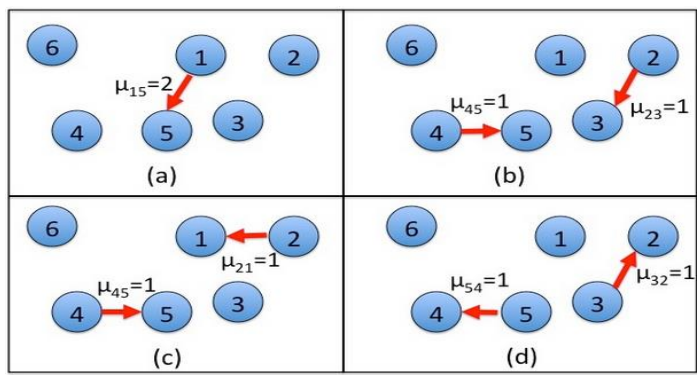

Fig.4.Topology for prioritization data [26] 


$$
\begin{array}{ll}
\mu_{a}=\left[\begin{array}{lllll}
0 & 0 & 0 & 2 & 0 \\
0 & 0 & 0 & 0 & 0 \\
0 & 0 & 0 & 0 & 0 \\
0 & 0 & 0 & 0 & 0 \\
0 & 0 & 0 & 0 & 0 \\
0 & 0 & 0 & 0 & 0
\end{array}\right], \quad \mu_{b}=\left[\begin{array}{lllll}
0 & 0 & 0 & 0 & 0 \\
0 & 0 & 0 & 0 & 0 \\
0 & 0 & 1 & 0 & 0 \\
0 & 0 & 0 & 1 & 0 \\
0 & 0 & 0 & 0 & 0 \\
0 & 0 & 0 & 0 & 0
\end{array}\right] \\
\mu_{c}=\left[\begin{array}{lllll}
0 & 0 & 0 & 2 & 0 \\
1 & 0 & 0 & 0 & 0 \\
0 & 0 & 0 & 0 & 0 \\
0 & 0 & 0 & 0 & 0 \\
0 & 0 & 0 & 1 & 0 \\
0 & 0 & 0 & 0 & 0
\end{array}\right], \quad \mu_{d}=\left[\begin{array}{lllll}
0 & 0 & 0 & 0 & 0 \\
0 & 0 & 0 & 0 & 0 \\
0 & 1 & 0 & 0 & 0 \\
0 & 0 & 0 & 0 & 0 \\
0 & 0 & 1 & 0 & 0 \\
0 & 0 & 0 & 0 & 0
\end{array}\right]
\end{array}
$$

Fig.5. Proposed topology matrix for prioritization data

It was observed that 6 nodes under these possibilities can neither send nor can receive. This may occur because the node 6 is already out of the boundaries of communication.

\section{G. Dynamic priority for service differentiation}

The network should make more efforts for delivering the packets that include real-time data with high priority. If two or several data with the same conditions exist in the output of the back-pressure algorithm or if the network is on the verge, dynamic prioritization will be used for service differentiation.

We offer two different models that support two different traffic classes: real-time traffic class, non-real-time traffic class.

Throughput and delay for the proposed model is as follows.

Equation [26]

$$
\begin{aligned}
& \text { Throu:RT }=>\text { Throu:NR } \\
& \text { Delay:RT }<=\text { Delay:NR }
\end{aligned}
$$

Each node sees its line's backlog and that of the neighboring nodes, each node sends data to the line whose buffering is less crowded than others, if the buffer of any node is not vacant, based on a dynamic prioritization for service differentiation, some data with real-time traffic stand voluntarily for sending, then it is time for data with non-real-time traffic.

If the buffer node is on the verge of congestion and data with real-time priority cannot be queued non realtime traffic data is deleted, so that the new entrant data, which are real-time data, will be in line with high priority.

Note that the decisions and selection of the optimized commodity in the back-pressure algorithm are true for dynamic prioritization algorithm to service differentiation, that is, if in a path the line's differential backlog is not compatible with the decisions and selection of optimized commodity in the back-pressure algorithm, then the dynamic prioritization algorithm for service differentiation for that path and data will not be applied.

\section{Simulation and Evaluation of the Proposed Model}

In this study, in order to introduce the method, according to the necessary research and review of several papers about simulation of software's (OPNET, OMNET, NS2, NS3) we came up with the conclusion the conclusion that the best simulator in terms of flexibility and efficiency is better than any aspect of NS2, with the same simulation being used. Parameter values being used in the network are in the table below. 
Table 1. Values of Parameters for simulation

\begin{tabular}{|c|c|}
\hline SIMULATION & NS2.34, NS2.35 \\
\hline Routing Protocols & AODV and TREE and Proposed Method \\
\hline Simulation Duration(sec) & $40,60,80,100,120$ \\
\hline Simulation area & $500 * 500 \mathrm{~m}$ \\
\hline Number of Nodes & $20,40,60,80,100$ \\
\hline Transmission Range & $10 \mathrm{~m}$ \\
\hline Movement Model & Chain Topology \\
\hline Mac Layer Protocol & 802.11 \\
\hline Queue & Drop tail \\
\hline Packet Rate & 4 packets/sec \\
\hline Traffic Type & CBR(UDP) \\
\hline Data payload & 512 Byte/packet \\
\hline
\end{tabular}

Fig 6. Packet delivery ratio

In Zigbee networks sending packets is usually achieved step by step or by several leaps. Packet delivery ratio in these networks is very important.

PDR is to the ratio of packet delivery which is obtained by dividing the sent packets by the number of packets received.

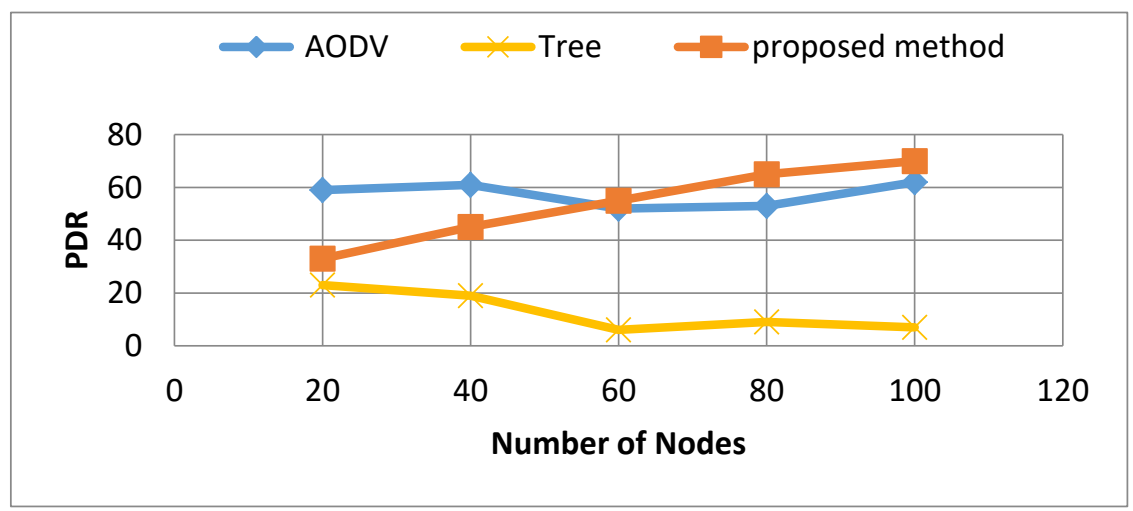

Fig.6. Figure related to PDR of the proposed algorithm

Healthy packet delivery ratio is conversely correlated with packet loss, when the network begins with twenty nodes, $30 \%$ of the packets are delivered safely and $70 \%$ are not, that is, $70 \%$ of the packets are lost. By increasing the number of nodes in the network and simulation time, delivery rate of healthy packets increases.

Fig 7. The number of Packet loss

Packet loss in the network can happen for various reasons, for example, packets to be sent over the network failed to reach the destination node, or they might be lost based on bit error or faulty hardware of the packet. The noise in the network can cause packet loss. Some packets loss in the network can be caused by traffic in this type of network. The packet delivery ratio is inversely proportional to the number of lost packets, that is, when we have $30 \%$ packet delivery ratio in 20 nodes, $70 \%$ of the packets has been lost in 20 nodes. 


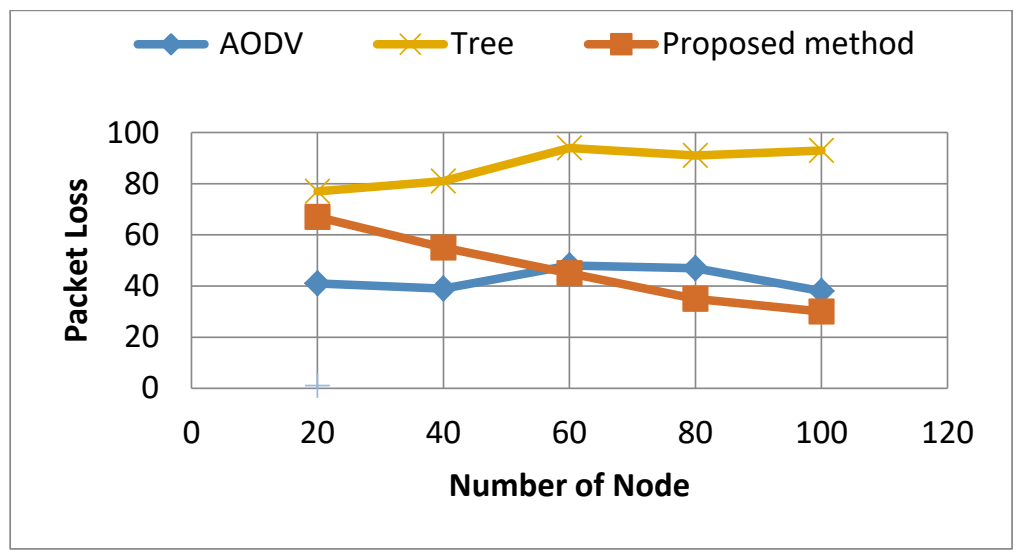

Fig.7. Figure related to packet loss in the proposed method

Fig 8. End-to-end delay

In ZigBee End-to-end delay refers to the time taken for a packet to be transmitted across a network from source to destination. This parameter is displayed with milliseconds. The proposed algorithm shows that it has better performance at different times and this argument indicates that the performance of the proposed model is better.

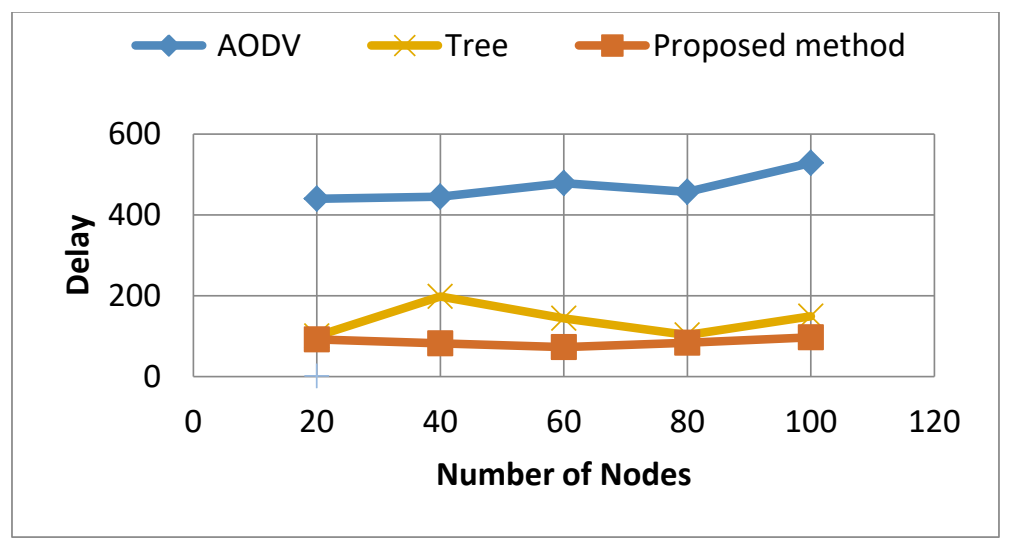

Fig.8. Figure related to the delay in the proposed method

Fig 9. Throughput

A network can be measured using different available tools on different operating systems. This page explains the theory according to which these tools are set for measurement and the issues related to this measurement. The reason for measuring the throughput of a network is that people often tend to know the maximum data throughput in a communication link or network access, in units of bits per second. The common method for measuring the quantity of this is that it sends a large file from one system to another, calculates the time required to complete the transfer or copy the files. Then throughput is obtained by dividing the file size by that time, in units of megabits per second, kilobits per second, or bytes per second. The following equation obtains the throughput in the network, which the throughput in wireless ad hoc networks is bits per second. 


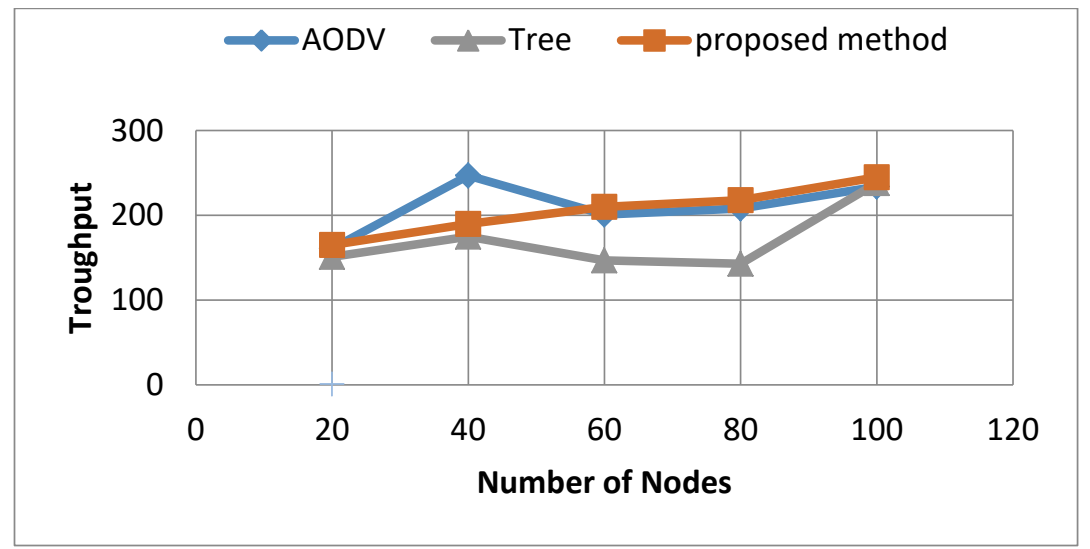

Fig.9. Figure related to the throughput of the proposed method

Fig 10. Normalization

In the first moment of simulation due to the high speed of movement of the nodes and unknown location of the nodes, routing does not happen properly in the network, over time, increasing the number of nodes, network routing is normalized and the routing is improved, which is commonly called network has been normalized.

In this simulation we have been successful in directing 16 packets out of 20 packets in 100 nodes, which the same number of packets in 20 nodes and at the beginning of the simulation explained above 4 packets were successfully directed, which has been improved compared to the tree routing.

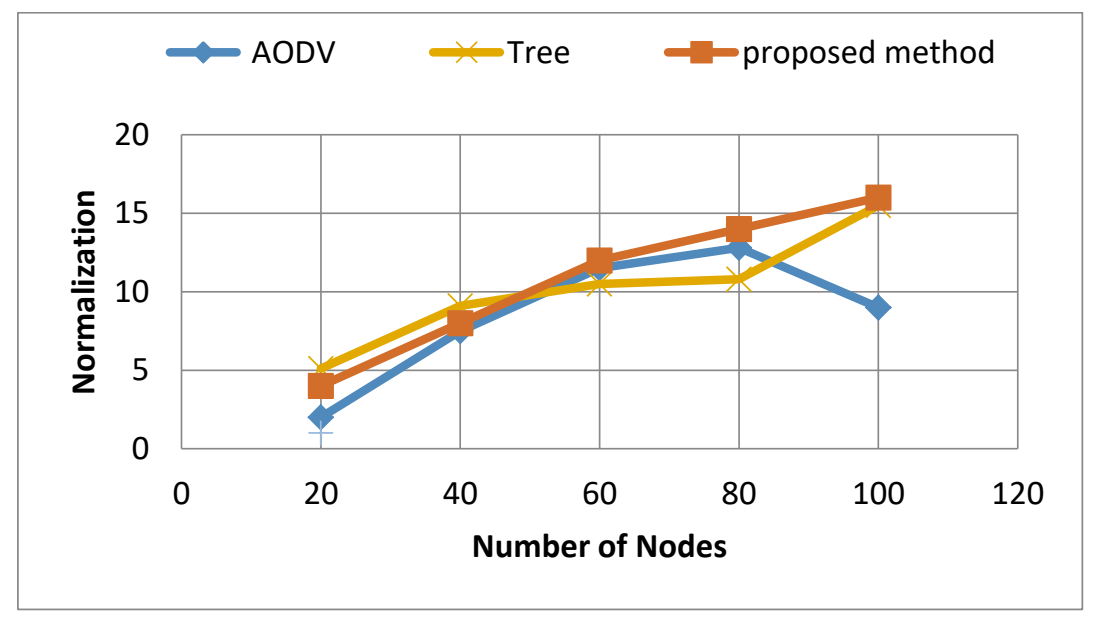

Fig.10. Figure related to normalization of the proposed algorithm

Fig 11. Mean Hop

The less we use hop in routing, the more efficient and secure the network becomes. This argument is because it is possible that along the path there may be corrupted hops, the less the number of the hops is, the lower the error rate becomes. Figure VI represents the lowest average number of hops used in the proposed model. 


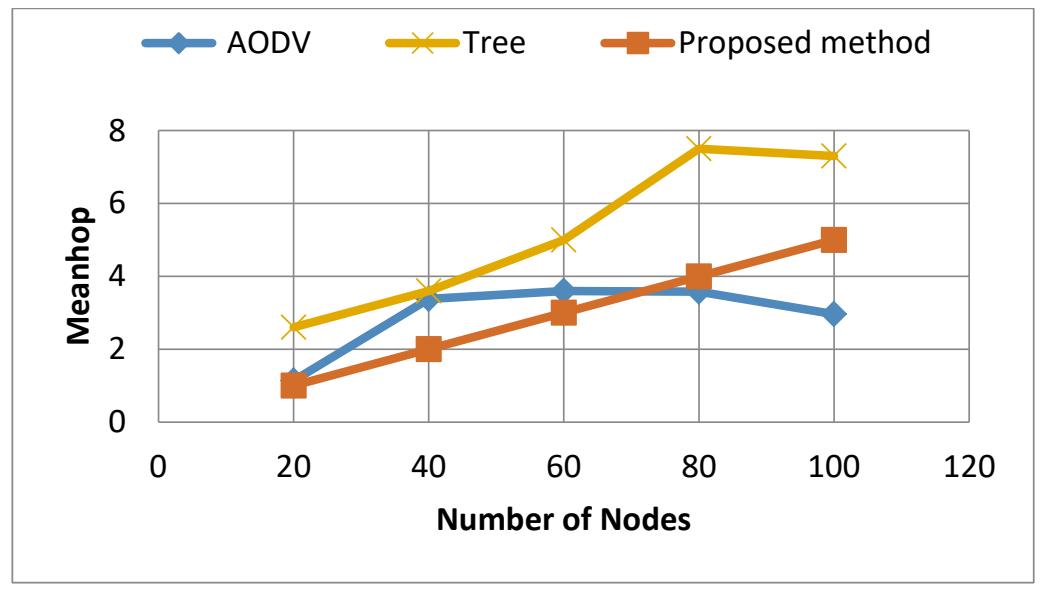

Fig.11. Figure related to hops in the proposed model

\section{Fig 12. Energy}

In this section, simulating the energy consumption of nodes that are connected to the thread due to routing and less steps, the remaining to the destination is used and this has helped to remain the energy of parent and main nodes and be used far better so that the network lifetime will not end earlier. However, these threading nodes, which are children and less-used, finish earlier; this would not pose any threat to our network. Below the energy consumption of nodes connected to the thread are presented in the proposed algorithm.

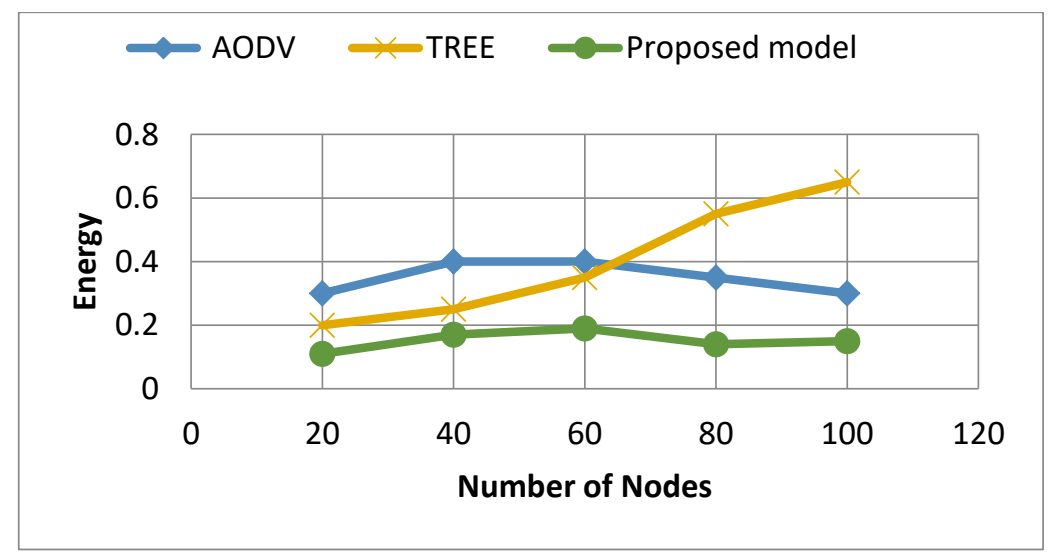

Fig.12. Figure related to energy consumption in the proposed algorithm

In this scheme, we have been inspired by the back-pressure algorithm through threading tree routing and have made changes to the standard tree protocol, which made improvements in the designed protocol compared to the standard tree protocol. With the removal of congestion to a great extent from the root by the proposed algorithm, we can refer to energy consumption in the main nodes.

\section{Conclusions}

In previous years, the tree routing protocol in this standard has been highly regarded due to the simplicity has been trying to reduce and fix its flaws. The algorithms defined in this context have focused on decreasing the 
number of leaps, which is one of the most important disadvantages of tree routing. Such algorithms, based on the selected shortcut are in search of nodes routes in the table of neighbourhood that provide a less number of leaps to reach the destination packet and thus have a significant role in reducing costs and delays of route, but what has been ignored in these algorithms is the balance of energy consumption in these nodes, which are the important points in ZigBee network. Since in the tree routing algorithm, each node is very important in the network due to the hierarchical nature of the algorithm and with the death of a node, the performance and lifespan of the network is affected; therefore, the energy balance plays an important role in the network lifetime and considering it in routing and optimal route selection to increase the lifetime of the network is inevitable. In this scheme, we were inspired by back-pressure algorithm through threading tree routing and made some changes to the standard tree protocol, which made improvements to the designed protocol compared to the standard tree protocol. With the removal of congestion to a great extent from the root by the proposed algorithm, we can refer to energy consumption in the main nodes. It seems that optimization of the bee and ant algorithms can be used for tree improvement and they can be designed in accordance with the algorithm. In addition, we can use other methods inspired by nature, including PSO, genetic algorithm, the fuzzy systems, nerve networks and so on to improve available protocols including tree protocol and new protocols with better performance can be offered.

\section{Reference}

[1] Akbar MAJIDI, Hamid MIRVAZIRI. BDCC: Backpressure routing and dynamic prioritization for congestion control in WMSNs. I.J. Computer Network and Information Security; 2014, 5, 29-34.

[2] Akbar MAJIDI, Hamid MIRVAZIRI. BDCC: Backpressure routing and dynamic prioritization for congestion control in WMSNs. I.J. Computer Network and Information Security; 2014, 5, 29-34.

[3] Ali C. Samuel P. 2014. A distributed energy-efficient clustering protocol for wireless sensor networks. Computers \& Electrical Engineering:Elsevier. 36: 303-312.

[4] D. Gislason, Zigbee Wireless Network, Newnes Publication Co., 2008.

[5] Banerjee T. Xie B. Jung HJ. Agrawal BD. 2010. Increasing lifetime of wireless sensor networks using controllable mobile cluster heads. 10: 313-336

[6] Basagni S. Carosi A. Melachrinoudis E. Petrioli C. Wang ZM. 2013. Controlled sink mobility for prolonging wireless sensor networks lifetime. Wireless Networks Jurnal:ACM. 17: 759-778.

[7] Boonsongsrikul A. Kocijancic S. Suppharangsan S. 2013. Effective energy consumption on wireless sensor networks: Survey and challenges .Information \& Communication Technology Electronics \& Microelectronics (MIPRO). 469-473.

[8] Chirag KR. Trilok CA. 2011. An improved transport layer protocol for wireless sensor networks. Computer Communications: Elsevier. 34: 758-764.

[9] Feng W. Jiangchuan L. 2011. Networked wireless sensor data collection: issues, challenges, and approaches. Communications Surveys \& Tutorials, IEEE. 13:673 - 687.

[10] Flora J. Kavitha DF. Muthuselvi V. 2011. A survey on congestion control techniques in Wireless Sensor Networks. Emerging Trends in Electrical and Computer Technology (ICETECT), International Conference on, 1146 - 1149.

[11] Network Simulator version 2.27 (ns-2.27): Available online, http://www.isi.edu/nsnam/ns/, March 2010

[12] J. Jheng, M.J.Lee ,'A comprehensive performance study of IEEE 802.15.4", Sensor network operations (IEEE Press, Wiley Interscience, 2006), Ch.4, pp. 218-237

[13] http://ees2cy.engr.ccny.cuny.edu/zheng/pub/, last access April 2008

[14] Hussaini M. Bello-Salau AF. Salami F. Anwar AH. Abdalla Md. Rafiqul I. 2012. Enhanced clustering routing protocol for power-efficient gathering in wireless sensor network. International Journal of Communication Network and Information Security(IJCNIS). 4: 18-28.

[15] Jenolin FD. Kavitha V. Muthuselvi M. 2011. A survey on congestion control techniques in Wireless Sensor Networks. Emerging Trends in Electrical and Computer Technology (ICETECT), 2011 
International Conference on. 1146-1149.

[16] Jing Z. Lei W. Suran L. Xiaokang L. Zhuxiu Y. Zhengjiu G. 2010. A survey of congestion control mechanisms in wireless sensor networks. Intelligent Information Hiding and Multimedia Signal Processing (IIH-MSP), 2010 Sixth International Conference on. 719-722.

[17] Karthik C. Sukumar R.Nageswari M. 2013. Sensors lifetime enhancement techniques in wireless sensor networks - A critical review. IRACST - International Journal of Computer Science and Information Technology \& Security.

[18] Kumari J. Prachi . 2015. A comprehensive survey of routing protocols in wireless sensor networks. Computing for Sustainable Global Development (INDIACom). 325-330.

[19] S.K. Singh, MP Singh, and DK Singh. Routing protocols in wireless sensor networks_a survey. International Journal of Computer science and engineering Survey (IJCSES), 1(2):63_83, 2010.

[20] S.C.Ergen, "Zigbee/IEEE 8.201504 Summary", Technical Report, September 2004.

[21] LIN QM. WANG RC. GUO J. SUN LJ. 2011.Novel congestion control approach in wireless multimedia sensor networks. The Journal of China Universities of Posts and Telecommunications:Elsevier. 18: 1-8.

[22] Lotf JJ. Nazhad, SHH. Alguliev RM. 2011. A survey of wireless sensor networks. Application of Information and Communication Technologies (AICT). 1-6.

[23] S.Ahn, D.Ko, B.Kim, S.Lee, 2013. "Energy-efficient Tree Routing Algorithm-based Destination Family Group in Zigbee Networks". Fourth International Conference on Sensor Technologies and Applications.

[24] Tashtarian F. Moghaddam MY. Effati S. 2012. Energy efficient data gathering algorithm in hierarchical wireless sensor networks with mobile sink. Computer and Knowledge Engineering (ICCKE). 232-237.

[25] Wei WF. Ji MC. Lei Sh. Tian Ch. De PQ. 2010. Congestion avoidance, detection and alleviation in wireless sensor networks. Journal of Zhejiang University SCIENCE. 11: 63-73.

\section{Authors' Profiles}

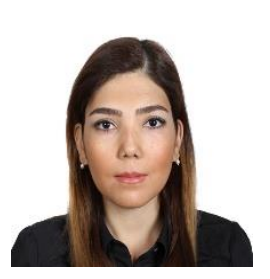

Negar Jadidkar received the B.S degree and Master degree in software and Computer Technology engineering and Computer architecture from Science and Research Islamic Azad University, Tehran, Iran. in 2014 and 2016 respectively her current research interests include wireless communications, Internet of think, and 5th generation of network.

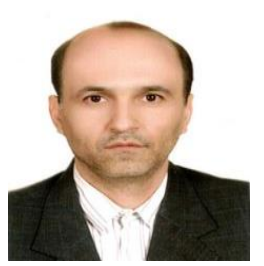

Hossein Samimi was born in Tehran-Iran, on May 7, 1972. He received the B.Sc. and M.Sc., degrees in electrical engineering from Tehran University, Tehran-Iran, in 1994 and 1998, respectively, and he did $\mathrm{PhD}$ in Electrical and Computer Engineering Department of Tarbiat Modarres University, Tehran- Iran. His current research interests include wireless communications, diversity systems, and digital communication over fading channels.

How to cite this paper: Negar Jadidkar, Hossein Samimi," Improvement of ZigBee Using by Thread and Backpressure Algorithm", International Journal of Wireless and Microwave Technologies(IJWMT), Vol.7, No.5, pp.1-13, 2017.DOI: 10.5815/ijwmt.2017.05.01 